\title{
Mini-gastric Bypass Complications and the Value of the Preoperative Neutrophil to Lymphocyte Ratio in Early Prediction of Complications
}

\author{
Fadli Dogan ${ }^{1}$ and Mürsit Dincer ${ }^{2}$ \\ ${ }^{1}$ Department of Surgery, Medical Park Hospital, Elazig, Turkey \\ ${ }^{2}$ Department of General Surgery, School of Medicine, Firat University, Turkey
}

\begin{abstract}
Objective: To describe the short-term results of mini-gastric bypass (MGB), the complications in patients who underwent mini-gastric bypass surgery (MGB), and evaluate the value of the neutrophil to lymphocyte ratio (NLR) in predicting complications at an early stage.

Study Design: A descriptive, analytical study.

Place and Duration of Study: Elazığ Medical Park Hospital, Elazığ, Turkey from January, 2016 to July 2019.

Methodology: Data of patients who had undergone MGB for morbid obesity at the Department of Surgery, Medical Park Hospital, Elazığ, Turkey from January 2016 to July 2019 were retrospectively evaluated. Demographic characteristics, preoperative body weight, body mass index (BMI), weight change after surgery, and postoperative complications were evaluated. The collected data were statistically analysed. The Kolmogorov-Smirnov and Shapiro-Wilk tests were used to evaluate the data distribution.

Results: A total of 95 patients were included in the study. The median preoperative BMI was $40.7 \mathrm{~kg} / \mathrm{m}^{2}(40.2-42.5)$, the median NLR was 2.1 (1.5-2.8), and the median length of hospital stay was 4.0 (3.0-4.0) days. In univariate analysis, age, gender, preoperative baseline BMI or preoperative NLR were not statistically significant related to the presence of postoperative complications. There was a statistically significant difference in BMI, depending on different measurement times during the follow-up period ( $p<0.001$ ). Conclusion: MGB is an effective procedure in obesity and diabetes surgery. Complications with high morbidity and mortality might be seen, although at low rates. Although NLR's efficacy is low in predicting MGB complications, it may be a useful parameter in a large series.
\end{abstract}

Key Words: Bariatric surgery, Complications, Mini-gastric bypass, Morbid obesity.

How to cite this article: Dogan F, Dincer M. Mini-gastric Bypass Complications and the Value of the Preoperative Neutrophil to Lymphocyte Ratio in Early Prediction of Complications. J Coll Physicians Surg Pak 2021; 31(01):70-73.

\section{INTRODUCTION}

Morbid obesity is a major health problem worldwide. ${ }^{1,2}$ The World Health Organization (WHO) has reported that at least 2 billion people are overweight globally. ${ }^{3}$ According to WHO data, in $2014,39 \%$ of adults were overweight, and $13 \%$ were morbid obesity. ${ }^{4}$ Obesity increases the risk of many systemic diseases and mortality. ${ }^{5}$ Due to failures in conservative treatment, surgery is becoming increasingly popular for the treatment of morbid obesity. The efficacy of laparoscopic Roux-en-Y gastric bypass (LRYGB) surgery, which has been performed for more than 50 years in obesity treatment, is undisputed.

Correspondence to: Dr. Mürsit Dincer, Department of General Surgery, School of Medicine, Firat University, Turkey

E-mail: drmursitdincer@gmail.com

Received: May 08, 2020; Revised: August 14, 2020;

Accepted: September 26, 2020

DOI: https://doi.org/10.29271/jcpsp.2021.01.70
However, even for experienced surgeons, the procedure presents some difficulties. ${ }^{1,3,6}$ Therefore, a surgical technique known as a single anastomosis gastric bypass or mini-gastric bypass (MGB) has been developed. ${ }^{3}$ In addition to having similar surgical results to LRYGB, MGB has some advantages, such as being a relatively simple technique and having a shorter learning curve for the surgeon and shorter surgery duration. ${ }^{5}$ Although LRYGB is accepted as the gold standard procedure in bariatric surgery, laparoscopic sleeve gastrectomy and MGB have been competitive with LRYGB in recent years. ${ }^{2,7,8}$

MGB is a single-anastomosis malabsorptive surgical technique preferred in morbidly obese patients. MGB is a safe and alternative procedure for LRYGB that has been demonstrated to be effective in weight loss and in resolving metabolic complications. ${ }^{2,4,6,9,10}$ The purpose of MGB is to enable a longer feeling offullness" after eating, both by reducing the stomach volume and by ensuring that foods are transferred to the terminal ileum faster with the modulation of intestinal hormones. Its advantages over LRYGB are that it is a single anastomosis and a relatively simpler procedure. However, it has several serious nega- 
tive aspects, including micronutrient deficiency and marginal ulcer gastrojejunal stenosis.

The neutrophil to lymphocyte ratio (NLR) is a parameter used as a safety marker in systemic inflammatory response. It is a helpful marker for determining the prognosis in many types of cancer as an indicator of inflammation. In this study, the purpose was to present the complications in patients who underwent MGB and to evaluate the value of NLR in predicting these complications atan early stage.

\section{METHODOLOGY}

The study was conducted retrospectively by scanning archives of patients diagnosed with morbid obesity at the Department of Surgery, Medical Park Hospital, Elazığ, Turkey from January 2016 to January 2019. Patients' record, who underwent MGB, were reviewed on the hospital database, and included consecutively in the study. The inclusion criteria were administration of MGB due to obesity and/or diabetes mellitus. The exclusion criteria were patients who underwent other metabolic surgery. The demographic characteristics of the patients, including age, height, weight, body mass index (BMI) and preoperative haemogram values, were recorded. The complications that occurred after surgery in the postoperative follow-up period were investigated. The NLR values of the patients, who had complications and those who had no complications, were compared; and the efficacy of the NLR in predicting these complications was determined. The surgeries were carried out by the same surgeon with the same technique. In the postoperative follow-up period, the standard follow-up protocol was applied to each patient. The patients who developed complications were ranked by the Clavien-Dindo classification.

The conditions of all the patients, who were diagnosed with morbid obesity and included in this study, were preoperatively evaluated. The patients in this study were preoperatively evaluated by a multidisciplinary group consisting of a dietitian, a psychiatrist and an endocrinologist. Operations were decided upon, if lifestyle changes and weight loss programmes were seen to be ineffective. A total of 95 patients with MGB were followed up and treated at the clinic within the specified time period. Verbal informed consent was obtained from all patients before the procedure. Ethics Committee approval was not necessary because of the retrospective study design. The study was conducted in accordance with the ethical principles put forward by the Helsinki Declaration.

Statistical analyses were performed using SPSS version 23 (IBM Corp., Armonk, NY, USA). The Kolmogorov-Smirnov and Shapiro-Wilk tests were used to evaluate the data distribution. The descriptive data are presented as frequencies (n) and percentages (\%) for categorical variables, mean with standard deviation for normally distributed numerical variables and median with interquartile range for non-normally distributed numerical variables. Univariate logistic regression analyses were performed to estimate the postoperative complication risk. Odds ratios and 95\% confidence intervals are presented for univariate logistic regression analyses. Mauchly's test of sphericity was used to determine the multivariate normality of the repeated measure data of the BMI. The related-samples Friedman two-way analysis of variance by rank test was used to compare the non-normal distributed data of the preoperative, postoperative 3-month and postoperative 6-month BMI measurements. Dunn's pairwise post-hoc test with Bonferroni correction was used for pairwise comparisons. A p-value $<0.05$ was considered statistically significant.

\section{RESULTS}

This longitudinal surgical study was conducted on patients with obesity admitted to the General Surgery Department, who underwent MGB surgery. A total of 95 patients were included in the study. The mean age was $49.7 \pm 9.8$ years; females comprised $36.8 \%$ of the patients. The median preoperative BMI was $40.7 \mathrm{Kg} / \mathrm{m}^{2}$ (40.2-42.5), the median NLR was 2.1 (1.5-2.8), and the median length of hospital stay was 4.0 (3.0-4.0) days.

By univariate analysis, gender, sex, preoperative BMI or preoperative NLR were not statistically significantly related to the presence of postoperative complications (Table I). There was a statistically significant difference in BMI depending on different measurement times during the follow-up period ( $p<0.001$ ). On pairwise comparison, statistically significant differences were found between preoperative and postoperative 3-month measurements, and preoperative and postoperative 6-month measurements ( $p<0.001$ and $p<0.001$, respectively). Complications developed in four cases in this study. According to the Clavien-Dindo classification, the Grade II case was the patient who received medical treatment due to biliary reflux and whose symptoms regressed after treatment. Two cases in Grade IIIb were operated again due to biliary reflux; and were converted into LRYGB. One case was operated due to anastomotic leakage in the early period.

\section{DISCUSSION}

Morbid obesity is a major health problem. ${ }^{1,2,11}$ Bariatric surgery procedures are becoming increasingly prominent in morbid obesity treatment due to inadequate conservative treatment and insufficient exercise / diet practices. ${ }^{12,13} \mathrm{MGB}$ is a single anastomosis malabsorptive procedure employed in bariatric surgery. When its short- and mid-term results are considered, it is found an effective method with acceptable weight loss, comorbidity and complication rates. ${ }^{1,2,4,10,14,15}$ However, serious complications such as biliary reflux, marginal ulcer and gastrojejunal stenosis might occur following MGB surgery. ${ }^{1,8}$ Although MGB resembles Billroth II surgery, it has superior characteristics in terms of biliary reflux. ${ }^{3}$ In MGB, the pouch is narrower and longer, which decreases biliary reflux. Another advantage is that anastomosis is carried out on the back wall of the pouch, which reduces biliary reflux by facilitating gastric emptying. ${ }^{3}$ The reported prevalence of biliary reflux following MGB is between $0.9 \%$ and $2.8 \%{ }^{1,16}$ In the present study, three patients had biliary reflux symptoms. Carbajo et al. prescribed $40 \mathrm{mg}$ of proton pump inhibitors and sucralfate for six months in patients who had biliary reflux symptoms. $^{1}$ 
Table I: Demographics and baseline characteristics of the patients.

\begin{tabular}{|l|c|c|}
\hline Variable & & \multicolumn{1}{c|}{$\begin{array}{c}\text { Odds ratio } \\
\text { (95\% confidence interval) }\end{array}$} \\
\hline Age (years) & $49.7 \pm 9.8$ & $1.01(0.91-1.12)$ \\
\hline Mean \pm SD & $35(36.8)$ & \\
\hline $\begin{array}{l}\text { Gender } \mathrm{n}(\%) \\
\text { Female } \\
\text { Male }\end{array}$ & $60(63.2)$ & \\
\hline $\begin{array}{l}\left.\text { Preoperative BMI (kg/m }{ }^{2}\right) \\
\text { Median (IQR) }\end{array}$ & $40.7(40.2-42.5)$ & \\
\hline $\begin{array}{l}\text { Neutrophil to lymphocyte ratio } \\
\text { Median (IQR) }\end{array}$ & $2.1(1.5-2.8)$ & \\
\hline $\begin{array}{l}\text { Length of Stay } \\
\text { Median (IQR) }\end{array}$ & $4.0(3.0-4.0)$ & \\
\hline Preoperative BMI & & \\
\hline Preoperative NLR & & \\
\hline SD: Standard deviation, IQR: Interquartile range; BMI: Body mass index, NLR: Neutrophil to lymphocyte ratio; *Univariate logistic regression analysis was used. \\
\hline
\end{tabular}

Here, the present authors also prescribed the similar treatment in the patients who had biliary reflux symptoms. In one case, the reflux symptoms were controlled after medical treatment. Two patients received a revision of RNY gastric bypass because of biliary reflux that was resistant to medical treatment.

Carbajo et al. reported that the major complications that required early-period reoperation were bleeding, leakage and obstruction. ${ }^{1}$ In this study, leakage was seen in an anastomotic line in one patient in the early postoperative period, and the patient underwent surgery again. The incidence of marginal ulcers following MGB surgeries ranges between $0.5 \%$ and $7.6 \%{ }^{1,7}$ In the present study, none of the patients who received MGB had a marginal ulcer in the follow-up period. Another important complication of MGB is anastomotic stenosis. Carbajo et al. reported stenosis at a rate of $0.5 \%,{ }^{1}$ and Kansou et al. reported a gastrojejunal stenosis rate of $16.9 \%{ }^{7}$ In this study, the longest follow-up period was 36 months, and no stenosis was detected in any patient.

Another important concern about the MGB technique is the probability of increased gastric and oesophageal cancer risk because of the biliary reflux. ${ }^{3}$ However, malignancy risk has not been shown to increase after MGB. ${ }^{14}$ In the study by Rutledge, which reported the results of the first 1274 MGB operations, the overall complication rate was $5.2 \%$; and the overall rates of deep vein thrombosis (DVP) and pulmonary embolism (PE) were $0.08 \%$ and $0.16 \%$, respectively. The leakage rate was determined to be $1.6 \%$. One patient died in hospital $(0.08 \%) .{ }^{16}$ Carbajo et al. reported the long-term complication rate as $1 \%$ in a series of 1200 cases. ${ }^{1}$ In the present study, the general complication rate was $1.5 \%$. No DVP or PE was detected. One patient was operated again at the sixth hour postoperatively because of anastomotic leakage. There was no mortality because of MGB. In his study, Rutledge gave multivitamins containing iron (15 mg), vitamin B12, folic acid, and vitamin D following MGB. ${ }^{16}$ In the present study, multivitamins were given to the patients in the postoperative period too, to avoid micronutrient deficiency.

NLR is a parameter used as a safety marker in systemic inflammatory response. It is an easy-to-obtain and cost-effective marker that is employed as an indicator of inflammation to help determine survival in many cancer types. ${ }^{17-19}$ NLR increases in patients who have higher tumour levels and nodal stages, and who have a higher number of metastatic lesions or more aggressive disease. ${ }^{17}$ In a study conducted by Halazun et al. to investigate the prognostic effects of NLR on 150 patients, who underwent liver transplantation because of hepatocellular carcinoma, higher incidence of recurrence and mortality was reported in patients who had higher NLR rates. ${ }^{18}$

In this study, it was not possible to determine that preoperative NLR was a strong predictor of complications and short hospital stays for MGB. It is thought that the situation occurred due to the small number of patients. In studies with larger patient series, it has been identified that enhanced NLR is associated with complications, which indicates that the NLR value can be used as an independent predictor of prognosis in MGB in a larger series study.

The limitations of this study were its retrospective design and limited number of cases. However, when the short-term results are considered, it is believed that the present study will contribute to the literature because it showed low complication rates and correct management of the complications.

\section{CONCLUSION}

MGB is an effective procedure in obesity and diabetes surgery. Complications that may lead to high morbidity and mortality might be seen, although at low rates. The NLR, which may be examined with whole blood count, is a parameter that can be easily employed because it has a low cost procedure and is easily accessible. Although its efficacy is low in predicting MGB complications in a large series, it may be a useful parameter.

\section{ETHICAL APPROVAL:}

Verbal informed consents were obtained from all patients before the procedure. Ethics Committee approval was not necessary because of retrospective study design. However, the study was conducted in accordance with the ethical prin- 
ciples put forward by the World Health Organization's Helsinki Declaration.

\section{CONFLICT OF INTEREST:}

Authors declared no conflict of interest.

\section{PATIENTS' CONSENT:}

As the study was designed retrospectively, data was collected from clinical archive.

\section{AUTHORS' CONTRIBUTION:}

FD, MD: Study design, data collection, literaure review, drafting of work, statistical analysis, final review, and approval of the article.

\section{REFERENCES}

1. Carbajo MA, Luque-de-León E, Jiménez JM, Solórzano JOD, Miranda MP, Alija MJC. Laparoscopic one-anastomosis gastric bypass: Technique, results, and long-term follow-up in 1200 patients. Obes Surg 2017; 27(5):1153-67. doi: 10.1007/ s11695-016-2428-1.

2. Kraljević M, Delko T, Köstler T, Osto E, Lutz T, Thommen S, et al. Laparoscopic roux-en-Y gastric bypass versus laparoscopic mini gastric bypass in the treatment of obesity: Study protocol for a randomised controlled trial. Trials 2017; 18(1):226. doi: 10.1186/s13063-017-1957-9.

3. Chaim EA, Ramos AC, Cazzo E. Mini-gastric bypass: description of the technique and preliminary results. Arq Bras Cir Dig 2017; 30(4):264-6. doi: 10.1590/0102-67202017 00040009.

4. Carbajo MA, Jiménez JM, Luque-de-León E, Cao MJ, López M, García S, et al. Evaluation of weight loss indicators and laparoscopic one-anastomosis gastric bypass outcomes. Sci Rep 2018; 8(1):1961. doi: 10.1038/s41598-018-20303-6.

5. Jamal W, Zagzoog MM, Sait SH, Alamoudi AO, 'ouf SA, Alghamdi AA, et al. Initial outcomes of one anastomosis gastric bypass at a single institution. Diabetes Metab syndr Obes 2019; 12:35-41. doi: 10.2147/DMSO.S180111.

6. Lee WJ, Yu PJ, Wang W, Chen TC, Wei PL, Huang MT, et al. Laparoscopic Roux-en-Y versus mini-gastric bypass for the treatment of morbid obesity: A prospective randomised controlled clinical trial. Annals of surgery 2005; 242(1): 20-28. doi: 10.1097/01.sla.0000167762.46568.98.

7. Kansou G, Lechaux D, Delarue J, Badic B, Gall ML, Guillerm $\mathrm{S}$, et al. Laparoscopic sleeve gastrectomy versus laparoscopic mini gastric bypass: One year outcomes. Int J Surg 2016; 33:18-22. doi: 10.1016/j.ijsu.2016.07.051.

8. Braghetto I, Csendes A. Single anastomosis gastric bypass (one anastomosis gastric bypass or mini gastric bypass): The experience with Billroth II must be considered and is a challenge for the next years. Arq Bras Cir Dig 2017; 30(4):267-71. doi: 10.1590/0102-6720201700040010.

9. Milone M, Lupoli R, Maietta P, Minno AD, Bianco P, Ambrosino $P$, et al. Lipid profile changes in patients undergoing bariatric surgery: A comparative study between sleeve gastrectomy and mini-gastric bypass. Int J Surg 2015; 14:28-32. doi: 10.1016/j.ijsu.2014.12.025.

10. Lee WJ, Ser KH, Lee YC, Tsou JJ, Chen SC, Chen JC. Laparoscopic Roux-en-Y vs. mini-gastric bypass for the treatment of morbid obesity: A 10-year experience. Obes Surg 2012; 22(12): 1827-34. doi: 10.1007/s11695- 012-0726-9.

11. Kang JH, Le QA. Effectiveness of bariatric surgical procedures. A systematic review and network meta-analysis of randomised controlled trials. Medicine 2017; 96(46):46 (e8632). doi: 10.1097/MD.0000000000008632.

12. Connelly JB, Duaso MJ, Butler G. A systematic review of controlled trials of interventions to prevent childhood obesity and overweight: A realistic synthesis of the evidence. Public Health 2007; 121(7):510-7. doi: 10.1016/j.puhe.2006.11.015.

13. O'brien PE, Sawyer SM, Laurie C, Brown WA, Skinner S, Veit $\mathrm{F}$, et al. Laparoscopic adjustable gastric banding in severely obese adolescents: A randomised trial. JAMA 2010; 303(6):519-26. doi: 10.1001/jama.2010.81.

14. Mahawar KK, Jennings N, Brown J, Gupta A, Balupuri S, K Small P. Mini gastric bypass: Systematic review of a controversial procedure. Obes Surg 2013; 23(11):1890-8. doi: 10.1007/s11695-013-1026-8.

15. Quan Y, Huang A, Ye M, Xu M, Zhuang B, Zhang $P$, et al. Efficacy of laparoscopic mini gastric bypass for obesity and type 2 diabetes mellitus: A systematic review and meta-analysis. Gastroenterol Res Pract 2015; 2015:152852. doi: $10.1155 / 2015 / 152852$.

16. Rutledge, R. The mini-gastric bypass: Experience with the first 1,274 cases. Obes Surg 2001; 11(3):276-80. doi: 10.1381/096089201321336584.

17. Guthrie GJ, Charles KA, Roxburgh CS, Horgan PG, McMillan DC, Clarke SJ. The systemic inflammation-based neutrophil lymphocyte ratio: Experience in patients with cancer. Crit Rev Oncol Hematol 2013; 88(1):218-230. doi: 10.1016/j.critrevonc.2013.03.010.

18. Halazun KJ, Hardy KJ, Rana AA, Woodland DC, Luyten EJ, Mahadev S, et al. Negative impact of neutrophil-lymphocyte ratio on outcome after liver transplantation for hepatocelIular carcinoma. Ann Surg 2009; 250(1):141-51. doi: 10.1097/SLA.0b013e3181a77e59.

19. Sato H, Tsubosa $Y$, Kawano T. Correlation between the pretherapeutic neutrophil to lymphocyte ratio and the pathologic response to neoadjuvant chemotherapy in patients with advanced esophageal cancer. World J Surg 2012; 36(3):617-22. doi: 10.1007/s00268-011-1411-1. 